\title{
A simple metric to quantify seismicity clustering
}

\author{
N. F. Cho ${ }^{1}$, K. F. Tiampo ${ }^{1}$, S. D. Mckinnon ${ }^{2}$, J. A. Vallejos ${ }^{2, *}$, W. Klein ${ }^{3}$, and R. Dominguez ${ }^{4}$ \\ ${ }^{1}$ Department of Earth Sciences, University of Western Ontario, London, Canada \\ ${ }^{2}$ Department of Mining Engineering, Queen's University, Kingston, Canada \\ ${ }^{3}$ Department of Physics, Boston University, Boston, USA \\ ${ }^{4}$ Department of Physics, Western Kentucky University, Bowling Green, USA \\ *now at: Department of Mining Engineering, University of Chile, Santiago, Chile
}

Received: 15 January 2010 - Revised: 10 May 2010 - Accepted: 14 May 2010 - Published: 2 July 2010

\begin{abstract}
The Thirulamai-Mountain (TM) metric was first developed to study ergodicity in fluids and glasses (Thirumalai and Mountain, 1993) using the concept of effective ergodicity, where a large but finite time interval is considered. Tiampo et al. (2007) employed the TM metric to earthquake systems to search for effective ergodic periods, which are considered to be metastable equilibrium states that are disrupted by large events. The physical meaning of the TM metric for seismicity is addressed here in terms of the clustering of earthquakes in both time and space for different sets of data. It is shown that the TM metric is highly dependent not only on spatial/temporal seismicity clustering, but on the past seismic activity of the region and the time intervals considered as well, and that saturation occurs over time, resulting in a lower sensitivity to local clustering. These results confirm that the TM metric can be used to quantify seismicity clustering from both spatial and temporal perspectives, in which the disruption of effective ergodic periods are caused by the agglomeration of events.
\end{abstract}

\section{Introduction}

A simple inspection of the distribution of hypocenters of earthquakes indicates that they do not occur randomly in space or time. Several attempts have been made in order to better understand the cause/consequence relationship between events (Reasenberg, 1985; Dieterich, 1994; Felzer et al., 2002; Baesi and Paczuski, 2004; Marsan and Lengliné, 2008; Zaliapin et al., 2008). Clusters of earthquakes are commonly addressed in terms of swarms, a set of events with no single predominant event, or sequences that are highly related to a mainshock of large magnitude.

\section{Correspondence to: N. F. Cho}

(ncho3@uwo.ca)
Mainshocks are often considered as stationary Poisson processes with a fixed occurrence rate over time (Kagan and Jackson, 1991) and the remainder of the associated seismicity is classified as foreshocks/aftershocks sequences. The definition of foreshocks and aftershocks is not absolute and the studies on seismicity clustering mentioned previously use different criteria to identify clusters. Kanamori (1981) considered different seismic patterns that can be used as precursors to large events, in which foreshocks are an important type, even though their occurrence is not mandatory prior to mainshocks. Mogi (1985) classified foreshock sequences into two types: C and D. In the first, the seismic activity increases gradually towards the mainshock. The opposite occurs in type D sequences, in which seismicity decreases towards the mainshock.

Aftershocks are an important source of information about the mechanism of earthquake triggering and they have been widely studied over the years. Mendonza and Hartzell (1988) studied the correlation between the spatial distribution of aftershocks and the coseismic slip in faults in California. The Epidemic Type Aftershock Sequence (ETAS) model (Ogata, 1988; Helmstetter and Sornette, 2002) was proposed to understand the occurrence of aftershocks as generated by a mainshock. Utsu (2002) assembled a series of studies on seismicity, including findings on the spatial distribution of aftershocks. Shcherbakov et al. (2005) studied the interoccurrence time interval between aftershocks using a nonhomogeneous Poissonian model.

In this work, the TM metric will be interpreted as a simple measure of clustering. This metric, originally developed to study liquid systems and glasses (Thirumalai et al., 1989; Thirumalai and Mountain, 1993), was applied to earthquake simulations (Ferguson et al., 1999) and to regional seismicity by Tiampo et al. $(2003,2007)$. The result was the identification of periods of metastable equilibrium in seismic activity. The relationship between the effective ergodic periods and certain types of seismicity patterns was also addressed

Published by Copernicus Publications on behalf of the European Geosciences Union and the American Geophysical Union. 
in Tiampo et al. (2010). These previous studies indicate that clustering plays an important role in the results. The interpretation presented here offers a clear understanding of how seismicity clustering affects the TM metric, especially for southern California.

This article begins with a demonstration of the framework in which the TM metric can be considered a measurement of seismicity clustering. The method is then applied to three different cases: synthetic data, the southern California dataset, and mining induced seismicity. Remarks and limitations of the method are considered in the conclusions.

\section{The Thirumalai-Mountain metric}

The ergodic hypothesis is an important assumption for classical statistical mechanics in order to relate micro and macro states (Farquhar, 1964; de Oliveira and Werlang, 2007). A classical definition of the ergodic hypothesis states that, for an ensemble of particles, the time average of a property $f(t)$ of a single particle and the ensemble average of the same property $f$ relate as follow

$\lim _{T \rightarrow \infty} \frac{1}{T} \int_{0}^{T} f(t)=\langle f\rangle$

where $\langle f\rangle$ is the ensemble average of $f(t)$.

The TM metric was first developed to study the effective ergodicity in liquids and glasses (Thirumalai et al., 1989; Thirumalai and Mountain, 1993). The same framework will be used in the present work to study seismicity. For a system comprised of $N$ particles and an observable $G$, the TM metric is written as

$\Omega_{G}(t)=\frac{1}{N} \sum_{j=1}^{N}\left[g_{j}(t)-\langle g\rangle\right]^{2}$

where $g_{j}$ is the time average of the observable $G$ for particle $j$ until $t$ and $\langle g\rangle$ is the average of $g_{j}$ over all particles $j$. Equation (2) is simply the spatial variance of the temporal mean of $g_{j}$. Effective ergodicity arises from the fact that the relationship between ensemble and particle time averages are addressed for a long but finite time interval to ensure that all the phase space is sampled with equal likelihood (Thirumalai and Mountain, 1993), and it occurs when $1 / \Omega_{G}$ is linear in time. If the time average of $g_{j}$ for a particle is the same as the ensemble average of $g_{j}$, the metric is null.

Tiampo et al. (2007) applied Eq. (2) to historic seismicity to identify periods of effective ergodicity by dividing the region of interest into a mesh of $N$ boxes. These are considered to be the particles of the system and the cumulative number of events per box $n_{j}$ was used as a proxy for the observable $G$ : the seismic energy released. It has been argued that out of the possible proxies available for the seismic released energy, the number of events displays the longest correlations in time (Jimenez et al., 2006).
Different magnitude cutoffs were considered in the search for effective ergodic periods in southern California and it was found that the metric changes considerably for each case (Tiampo et al., 2007). The lack of effective ergodic periods for smaller events was hypothesized to be due to the stability of the catalog of southern California for the lower magnitude range. The system is stationary during these periods of effective ergodicity, meaning that the average of the studied property is constant over the considered period, and can be considered in a state of metastable equilibrium (Tiampo et al., 2003). Metastable equilibrium is a state that the system tends to occupy unless a disturbance is strong enough to propel the system to a new, but more stable, state.

Equation (2) can be re-written in terms of the variance of the cumulative number of events in each box $n_{j}(t)$. For simplicity, a simple translation in time is considered so that the initial time considered $t_{0}$ is set to be zero and $\Delta t=t_{\mathrm{f}}-t_{0}=t$. The TM metric then becomes

$$
\begin{aligned}
\Omega_{n}(t) & =\frac{1}{\Delta t^{2}} \frac{\sum_{j}^{N}\left(n_{j}(t)\right)^{2}}{N}-\frac{1}{\Delta t^{2}}\left(\frac{\sum_{j}^{N} n_{j}(t)}{N}\right)^{2} \\
& =\frac{1}{t^{2}}\left(\left\langle n_{j}(t)^{2}\right\rangle-\left\langle n_{j}(t)\right\rangle^{2}\right) .
\end{aligned}
$$

Periods in which the inverse TM metric is linear with a positive slope, $\frac{1}{\Omega_{n}(t)}=\frac{t}{D_{\mathrm{e}}}$, are considered to be effective ergodic and in a state of metastable equilibrium. From the latter and Eq. (3), it can be seen that the variance of $n_{j}(t)$ is linear in time during the effective ergodic periods. This can be interpreted as the variance of a normal diffusion processes, and the parameter $D_{\mathrm{e}}$ can be regarded as a diffusion parameter (Tiampo et al., 2007) related to the rate in which the phase space is sampled. In this case, $D_{\mathrm{e}}=\frac{1}{\Omega_{n}\left(t_{0}\right)}$ where $t_{0}$ is the initial time considered.

Equation (3) can be examined to analyze the effects of clustering in the TM metric. Its rightmost term is proportional to the square of the sum of cumulative events per box. It is sensitive to variations in the total number of events in each time step and thus it can be used to quantify temporal clustering of events. The same cannot be said for spatial clustering: different spatial configurations of a fixed number of hypocenters will yield the same sum of cumulative events. The left term in the right side of Eq. (3) can be regarded as a more complete measurement of clustering (refer to Appendix A).

A simpler form of the TM metric can be obtained if $\Sigma n_{j} \ll N$ or $\Sigma n_{j} \ll \Sigma n_{j}^{2}$. Under one of these assumptions, the TM metric can be written as

$\Omega_{n}(t)=\frac{1}{t^{2}}\left\langle n_{j}(t)^{2}\right\rangle$

and considering the scaling in time of the TM metric for ergodic periods,

$$
\begin{aligned}
\Omega_{n}(t) & \propto t^{-1} \\
\left\langle n_{j}(t)^{2}\right\rangle & \propto t .
\end{aligned}
$$



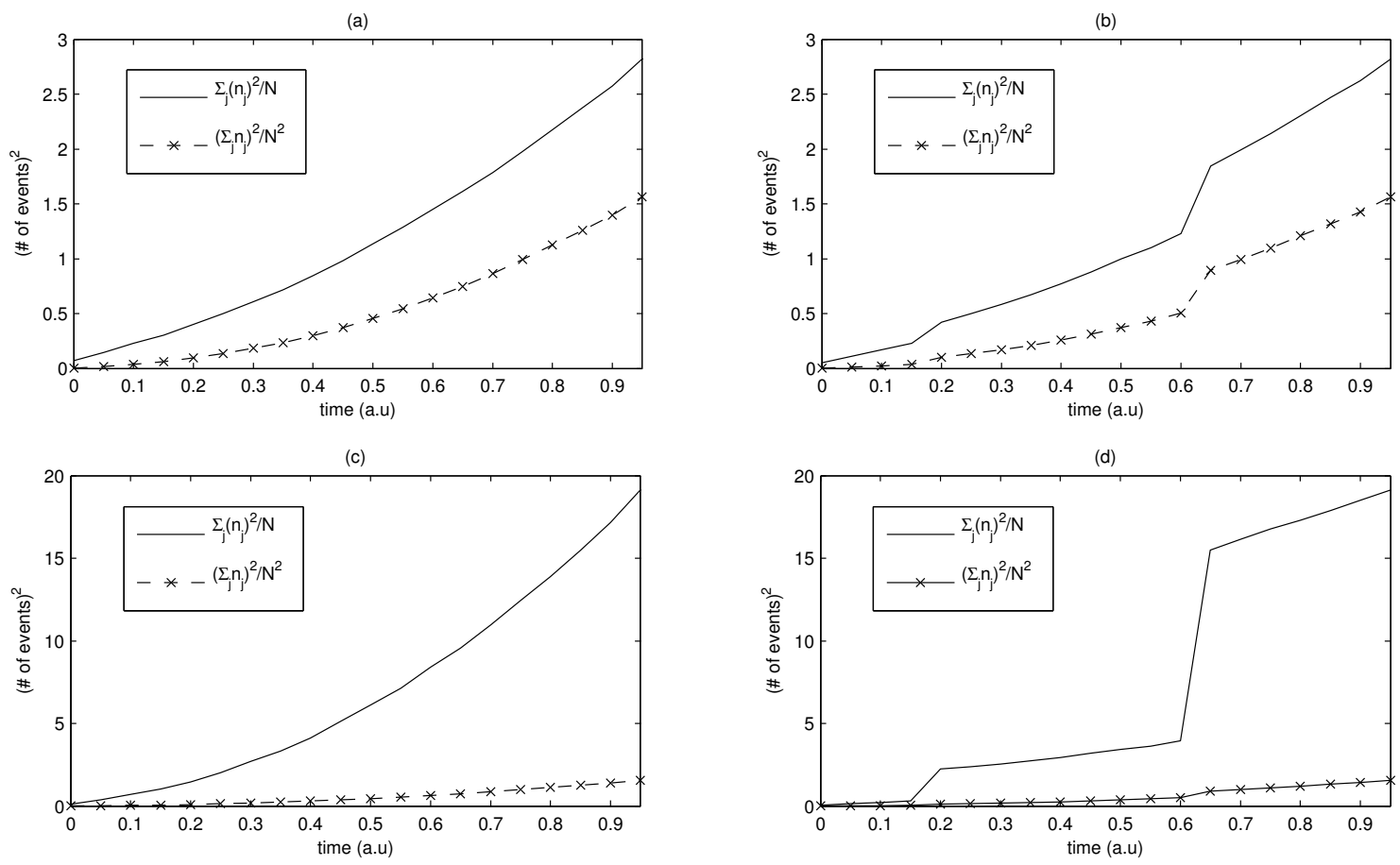

Fig. 1. Plot of the two terms in Eq. (3) for four different cases. Figure 1a represents the case with 10000 events randomly distributed in time and space, Fig. 1b obtained from clustering 600 and 1500 out of the 10000 events at $t=0.2$ and 0.7 , respectively, Fig. 1c is obtained by clustering 600 and 1500 out of the 10000 events spatially with the same latitudes and longitudes and Fig. 1d is obtained from the combination of the latter 2 scenarios.

The imposed linearity in time for the effective ergodic periods can be regarded as a benchmark to compare the evolution of seismicity clustering in time. It is important to stress, however, that the values obtained by the metric are non-unique. This means that different configurations might yield the same result.

Techniques that identify seismicity pattern changes often measure variations relative to long-term averages and generally are more accurate during ergodic periods because the spatial and temporal averages are stationary and approach the same value. Tiampo et al. (2010) showed that a better effectiveness of the Pattern Informatics (Tiampo et al., 2002; Holliday et al., 2006), a method that quantifies seismicity changes, was achieved during effective ergodic periods. The interpretation of effective ergodic periods presented here is similar to the relationship between the time evolution of the metric for liquids and systems in thermal equilibrium (Mountain and Thirumalai, 1989). In this analogy, the temperature of the system is the background seismicity and noise when dealing with the cumulative number of events and these effective ergodic periods are disrupted by the aftershock sequences from large earthquakes.

\section{Results}

\subsection{Synthetic catalog}

The method was initially tested for a simple synthetic catalog comprised of 10000 recordings for different scenarios. These scenarios are generated by distributing events both randomly and artificially clustered in space and time. Temporal and spatial coordinates were distributed in a unity interval for simplicity. The first case consists in a catalog comprised of 10000 events randomly displaced in space and time. A time clustering scenario was achieved for the second case by agglomerating 600 and 1500 events of the initial 10000 for times $t_{1}=0.2$ and $t_{2}=0.7$, respectively. For the third case, spatial clustering was obtained by giving the same latitudes and longitudes to subset of 600 and 1500 events while maintaining their randomness in time. The last case is obtained by combining the previous two scenarios into one where the clustered subsets partially overlap to produce clusters in space, time, and both.

The initial step is to verify the feasibility of the approximation for the TM metric illustrated in Eq. (5). Figure 1 displays both terms used to compute the full form of the TM metric in Eq. (3) for all scenarios. The random and time clustered cases are displayed in Fig. 1a and b, respectively. In these cases, Eq. (5) does not approximate the TM metric since $\left(\Sigma n_{j}\right)^{2}$ is not negligible compared to $\Sigma n_{j}^{2}$. Figure 1c illustrates the scenario with spatial clustering alone and it 


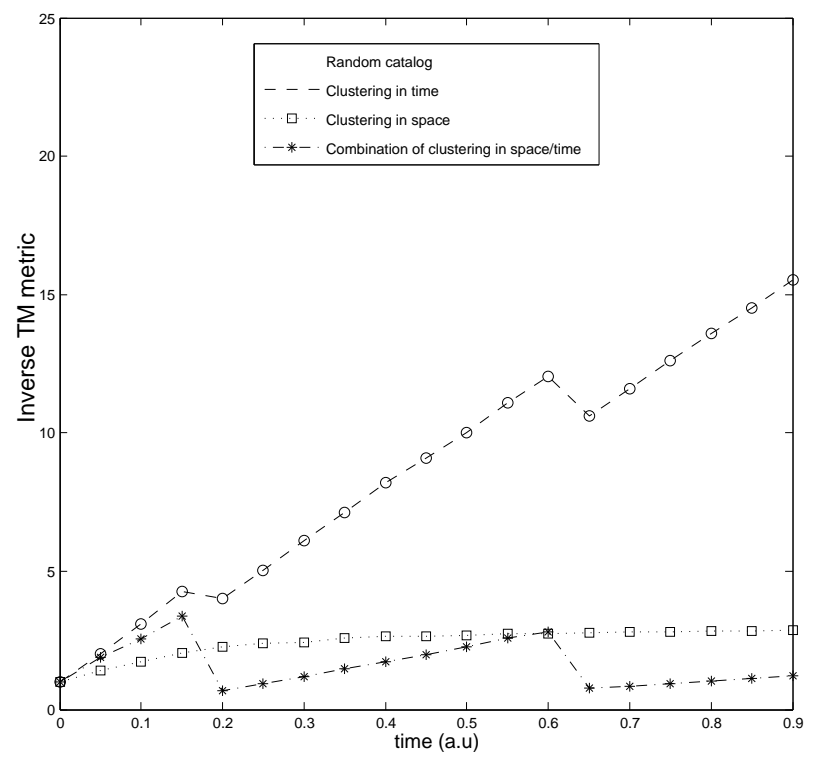

Fig. 2. Inverse TM metric for the different scenarios described in Fig. 1. From the uppermost to the lowermost solid line: random catalog, temporal clustering only, spatial clustering only, and the combination of both temporal and spatial clustering.

shows that $\left(\Sigma n_{j}\right)^{2}$ remains the same compared to the random catalog case in Fig. 1a, whereas $\Sigma n_{j}^{2}$ increases considerably for all $t$. This demonstrates that the first term does not measure spatial clustering, as noted previously, and that the approximation in Eq. (5) becomes feasible in the presence of spatial clustering. The same rationale can be applied to the last scenario illustrated in Fig. 1d, in which temporal and spatial clustering are considered simultaneously, to validate the applicability of Eq. (5). In this case, $\left(\Sigma n_{j}\right)^{2}$ is the same as in Fig. 1b while $\Sigma n_{j}^{2}$ increases substantially for all $t$ due to the spatial clustering.

Both terms from Eq. (3) display a non-linear behavior in Fig. 1a, scaling as $t^{\beta}$ and $t^{\alpha}$ where $\beta, \alpha>1$. Their combination, however, results in the linear trend of the inverse TM metric along the entire time domain observed for this case in Fig. 2. An examination of Eq. (3) and the linearity of the inverse TM metric indicate that the cumulative number of events per box $n_{j}$ evolves as a normal diffusive process during this long effective ergodic period. Based on the interpretation of Tiampo et al. (2007), the period in which the inverse TM metric is linear is effective ergodic. The original work on the TM metric in glasses and liquids relates effective ergodicity and fluids in thermal equilibrium (Mountain and Thirumalai, 1989). Considering the cumulative number of events per box $n_{j}$ as a proxy for the seismic released energy and the latter statement, it can be inferred that effective ergodic periods result from configurations in which the number of events are evenly distributed over the boxes. This suggests that these periods are characterized by non-clustered seismic activity.
Time clustering is verified from the abrupt vertical shifts of both $\left(\Sigma n_{j}\right)^{2}$ and $\Sigma n_{j}^{2}$ during the instances when the imposed agglomerations occur in Fig. $1 \mathrm{~b}$ and c. As discussed previously, both terms of Eq. (3) respond to the introduced temporal clustering. Figure 2 shows that the effective ergodic period displayed throughout the whole time domain for the random catalog in both time and space is disrupted during the instances when the time clustering is introduced. In between the instances when the temporal clustering is inserted, the system is in an effective ergodic state. The imposed temporal clustering in the given instants $t_{1}$ and $t_{2}$ promotes abrupt changes in the rates of seismic activity which translates to a sudden increase of the TM metric during these instances. This may be interpreted as a break in the thermal equilibrium of the system while the disruptions in the seismicity rate lasts, which means changes of the phase space subset where the system resides.

Figure 1c shows that the spatial clustering alone changes the scaling of $\Sigma n_{j}^{2}$ to $t^{\beta^{\prime}}$ while maintaining the same $t^{\alpha}$ scaling for $\left(\Sigma n_{j}\right)^{2}$ observed for the random case, where $\beta^{\prime}>\beta>\alpha$. This is not a localized effect as observed for time clustering, but rather a global effect that is spread over the entire period considered. By assuming the approximation for the variance of $n_{j}(t)$ to be $\left(\Sigma n_{j}\right)^{2}$ and its scaling with time shown in Fig. 1c, it can be inferred that the cumulative number of events per time step evolves in time as a super-diffusive process. The latter differs from the normallike diffusive behavior observed for effective ergodic periods and the result is the smooth non-linear trend observed for the inverse TM metric of the spatially clustered case in Fig. 2. The Coso Geothermal Field in southeastern California is an example of a region where seismicity occurs in a swarm-like manner and might display spatial clustering with little-to-no temporal clustering (Lees, 1998).

A more realistic situation encompasses both time and space clustering. The previous individual analysis of the effects of time and space agglomeration allow for the study of their combination and the results are also illustrated in Figs. 1 and 2. The vertical shifts observed due to time clustering are enhanced for $\Sigma n_{j}^{2}$ as $t$ increases due to the spatial clustering effects. The combination of the super-diffusive nature of $n_{j}(t)$ due to spatial clustering and the change in the scanned phase space subset generated by time clustering results in a system that is locally effectively ergodic. The term "locally" means that the system is effectively ergodic within the period during which temporal clustering does not occur: whenever $t \neq t_{1}$ and $t \neq t_{2}$. Once it happens, the system is reset and a different normal diffusion process takes place. This behaviour is illustrated in Fig. 2: from the moment when each of the two time clustering occurs, the inverse TM metric displays effective ergodic periods with different diffusion coefficients. 


\subsection{Southern California}

Implementing the method to synthetic catalogs offers a good foundation to understand the effects of different seismicity patterns to Eqs. (4) and (5). However, real seismicity offers a behavior which is intrinsically more complex and a deeper understanding of the technique is required. The next step is to apply the method to a well studied dataset: the southern Californian catalog. The spatial clustering in this data allows for the application of the approximation in Eq. (5) as discussed previously.

Tiampo et al. (2007) showed that events recorded from 1932 to 2006 in southern California with magnitude $M 4$ or greater display long effective ergodic periods that are disrupted by large events. These periods of effective ergodicity were disrupted by some large earthquakes, but not all. The 1952 Kern County, the 1979 Imperial Valley and the 1992 Landers earthquakes but not the 1989 Loma Prieta disrupted the effective ergodic period.

The same set of data is used with this method to better understand the previous results in terms of seismicity clustering. A mesh of $0.1^{\circ} \times 0.1^{\circ}$ is considered for the region between latitudes $32^{\circ} \mathrm{S}$ and $40^{\circ} \mathrm{S}$ and longitudes $-115^{\circ}$ and $-125^{\circ}$. Figure 3 shows the plot of Eq. (4) for the data considered. The dashed lines identify the large events that disrupt the effective ergodic periods: the 1952 Kern County, the 1979 Imperial Valley, the 1992 Landers, and the 1999 Hector Mine earthquakes. A vertical jump is observed for the years in which these events occurred. The exception is the 1979 Imperial Valley event, due to its late occurrence in the year (October), so that the bulk of the disruption occurs in the following year. It is important to stress that no premonitory pattern can be obtained from Fig. 3 due to the scale of the temporal discretization of the system: the effects of foreshocks/quiescence/aftershocks are all combined into one when considering a yearly time-discretization for the system.

Interesting features can be observed in Fig. 3. The first is the constant decrease in the amplitude of the vertical jumps as the years pass. This is attributed to the saturation of the cumulative number of events per box over time, which makes the effects of clustering less pronounced over the years due to the accrual of larger numbers events over the entire region and the asymptotic behavior of $\Omega_{n} \rightarrow \infty$ as $t \rightarrow \infty$. This illustrates importance of the choice of $t_{0}$ : the later $t_{0}$ is, the larger the response for the 1992 Landers and 1999 Hector mine earthquakes. This result also was noted by Tiampo et al. (2007). Here, this saturation results in the method not being able to detect the clustering due to the 1989 Loma Prieta earthquake.

The second important feature observed in Fig. 3 is the change in the slope of the linear regressions obtained with a Pearson's correlation coefficient greater than 0.97 between large events: from 1933 to 1951, from 1955 to 1978 , and from 1980 to 1991. As mentioned previously, these periods are effective ergodic and they correspond to intervals of time

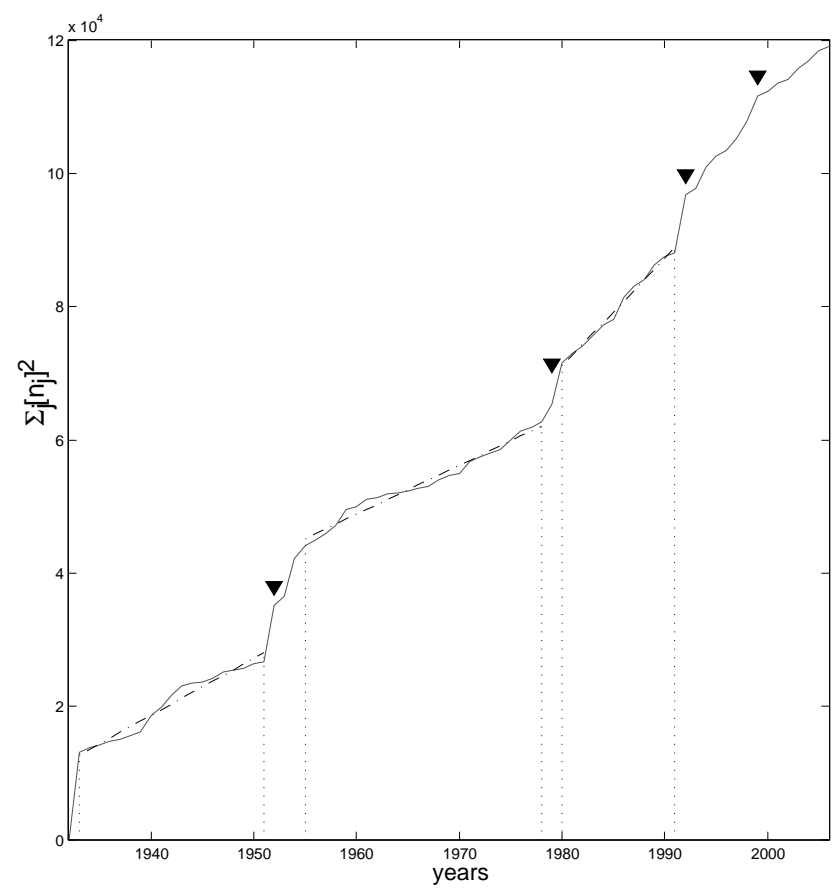

Fig. 3. Plot of Eq. (4) for the southern California dataset from 1932 to 2006. Dash-dot lines are linear regressions for the three effective ergodic periods from 1933 to 1951, from 1955 to 1978, and from 1980 to 1991 . Upside triangles locate the years of the occurrences of large earthquakes: the 1952 Kern County, the 1979 Imperial Valley, the 1992 Landers and the 1999 Hector Mine.

in which the system displays a normal diffusive behaviour. The different slopes indicate that these diffusive processes sample the different subsets of the phase space at different rates. Additional work remains to determine whether this apparent rate change is due to a sampling effect from changes (primarily increases) in the seismic network, local and/or regional effects related to changes in the stress field from the large events themselves combined with tectonic and geologic heterogeneities, or some combination thereof.

Figures 4 to 6 display the distribution of the cumulative number of events prior to, along with the number of events before and after the 1952 Kern County, the 1999 Landers and the 1989 Loma Prieta Earthquakes. The cumulative activity prior to the 1952 Kern County event is not considerably larger than the activity following the mainshock. As a result, the computation of Eq. (4) for $t=1952$ leads to the considerable vertical jump observed in Fig. 3. The effects of the increase in the cumulative number of events per box can be seen for the 1999 Landers earthquake illustrated in Fig. 5: the seismic activity after this event occurred in a region of considerable historic activity. The 1989 Loma Prieta earthquake, as displayed in Fig. 6, occurred in a region where the cumulative number of events prior to 1989 was considerably lower than the rest of the map. As a result, the clustering of seismic activity after this mainshock was not enough to 

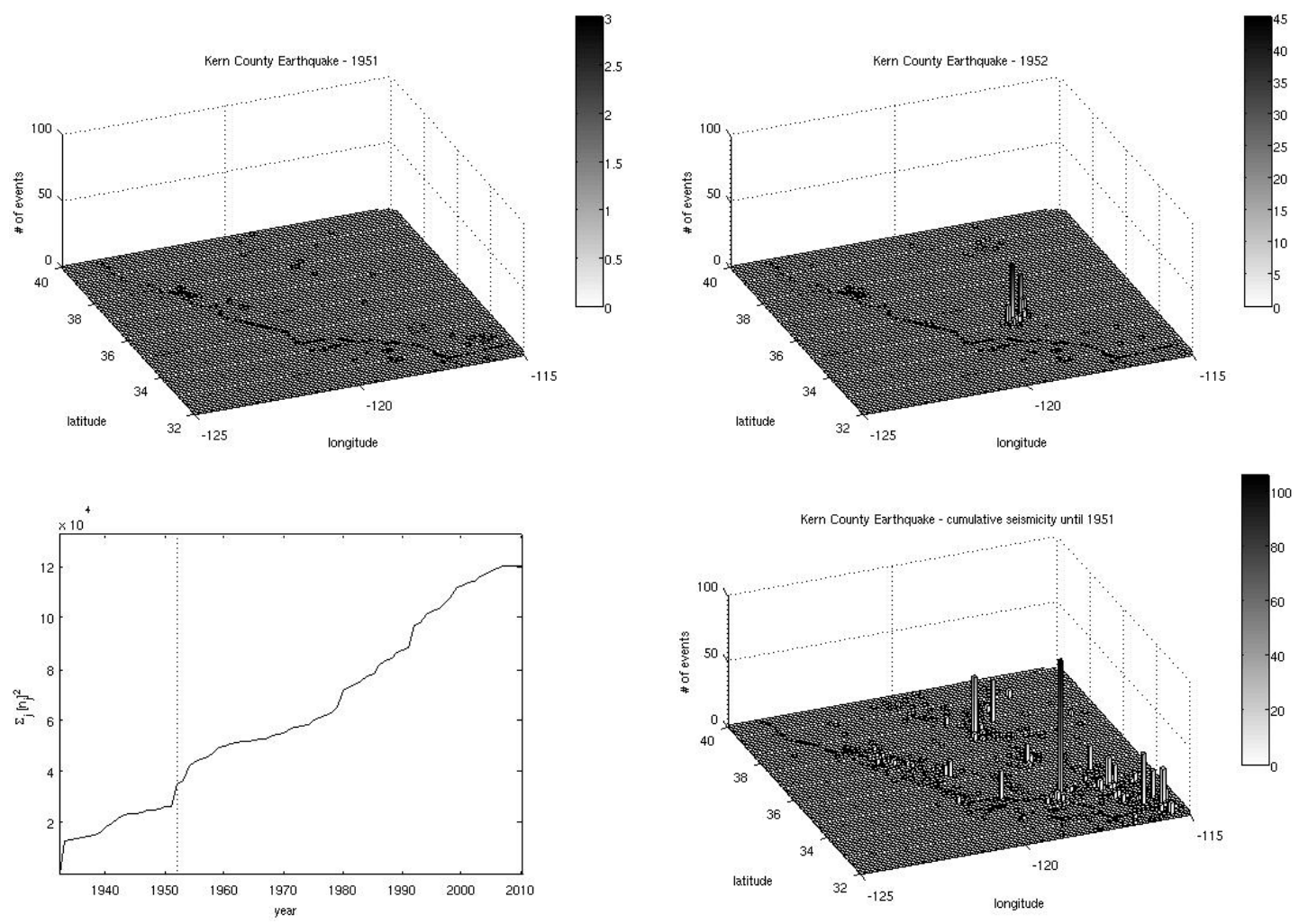

Fig. 4. Distribution of events before the 1952 Kern County earthquake before the mainshock (top left), after the mainshock (top right) and up until the mainshock since 1932 (bottom right). The approximation in Eq. (4) with the time of occurrence of the 1952 Kern County event as the dashed line are also plotted (bottom left).

promote a large disruption in the evaluation of Eq. (4). The latter can be attributed to the differences in seismic activity between the northern and southern California.

\subsection{Mining seismicity}

Mining induced seismicity (MIS) represents an interesting source of information due to the range of magnitudes that are involved: between lab controlled experiments and crustal seismicity. It can then offer important information on the scaling laws of seismicity and the nature of earthquake triggering. Economical factors also play an important role and, as a result, this topic has been extensively studied (Gibowicz and Kijko, 1994; Richardson and Jordan, 2002).

As a result, this method was also applied to MIS from two mines in Ontario, Canada. The dataset from Kidd Creek D Mine was obtained from August 2004 to May 2007 and it consists of 23000 event recordings. For Macassa Mine, over 10000 events were recorded from December 2004 to May 2007. A 3-D version of the method was used and different space/time configurations were tested. The outcomes displayed similar behaviours and, in the present work, only the results from cubes with an edge length of $10 \mathrm{~m}$ and a time binning of 7 consecutive days are shown for both mines.
Blasting activity is the main mechanism that drives the occurrence of small earthquakes in mines. As a result, MIS display a bimodal nature: small events highly clustered in time or space that are created by the blasts and larger, tectonic-like seismicity. Different bimodal distributions are constantly used to describe MIS (Gibowicz and Kijko, 1994). Richardson and Jordan (2002) used a set of simple criteria based on the space/time distance between events to identify the highly clustered blasting related events.

From the results obtained so far with this method, significant variability of Eq. (4) is expected. Its plot along with the seismic activity for Kidd Creek D is illustrated in Fig. 7. Frequent low-magnitude blasting activity generates most of the clustering observed in the various discontinuities in the plot. Note the horizontal feature in Fig. 7 around the period between the 100th and 140th weeks. The drop in the seismicity rate observed in Fig. 7 and the change of blasting sites are the reasons for the constant values of Eq. (4). The first automatically decreases the values obtained for Eq. (4) whereas the second has a more substantial role in the observed feature. As mentioned previously for the 1989 Loma Prieta earthquake, the method is unable to detect clustering in areas with a considerably lower seismic activity compared 

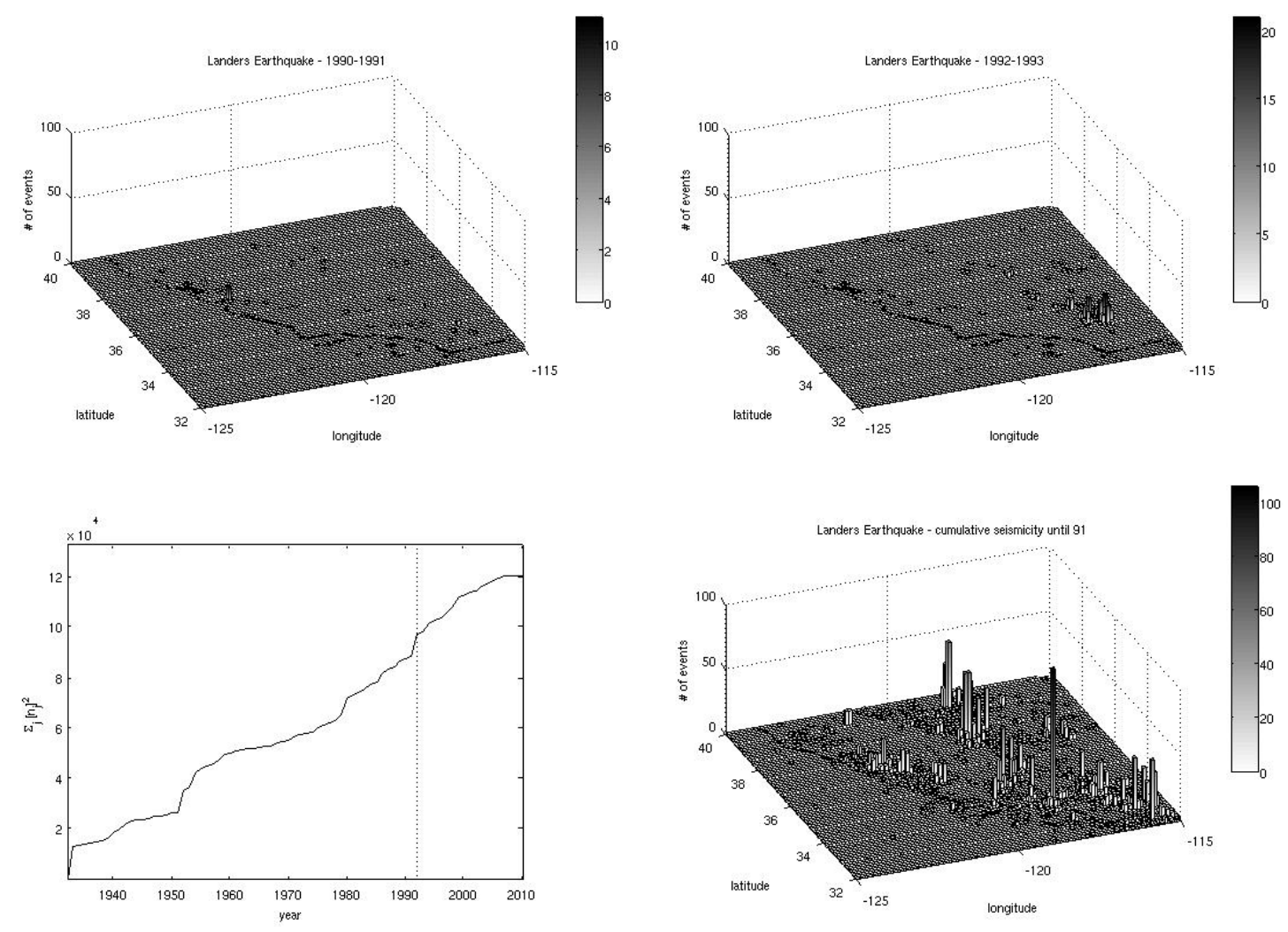

Fig. 5. Distribution of events before the 1992 Landers earthquake before the mainshock (top left), after the mainshock (top right) and up until the mainshock since 1932 (bottom right). The approximation in Eq. (4) with the time of occurrence of the 1992 Landers event as the dashed line are also plotted (bottom left).

to the rest of the region. During the instances in which $\Sigma n_{j}^{2}$ is constant, the blasting activity was shifted to regions with no or low previous seismic activity.

For Macassa Mine, the result of the method is plotted in Fig. 8. As indicated for the previous mine, two plateaus in the values of Eq. (4) are observed and they coincide with periods of low seismic activity. This mine displayed a completely different blasting regime compared to Kidd Creek D: rare high energy blasts. During the first period, around the 80th week, there was a shift in the blasting sites followed by a stoppage in the blasting activities. The second period around the 110th week is highlighted by a complete halt in the blasting process resulting in the sudden drop in seismic activity, leading to a second plateau. Once again, the historic seismicity played an important role as observed for the previous mine and southern California.

\section{Conclusions}

The TM metric is a simple metric that was first applied to study glass and liquid systems. Tiampo et al. (2007) showed that this metric can be used to identify periods of effective ergodicity, in which the system is considered to be in a metastable equilibrium state as a gas in thermal equilibrium, and that seismicity clustering seems to play an important role in this framework. These effective ergodic periods are interpreted as periods of time in which the evolution of cumulative number of events per box behave as a normal diffusive process. It is shown that the metric and the phenomena observed in the previous work can be attributed to seismic clustering and that, under the right assumptions, this metric can be simplified. Spatial clustering in seismicity allows for an approximation to the metric in which the effects of clustering in both time and space are simpler to account for.

While the determination of effective ergodic periods which ensure that spatial and temporal averages are stationary and confident is important for seismic hazard analysis, here we investigated the insight gained into seismicity clustering from the behaviour of the metric under various spatial and temporal end members clustering models. This interpretation was tested for three sets of data: synthetic, the southern California dataset and mining induced seismicity. From the synthetic data, it was observed that the effects of spatial and temporal clustering are of a different nature. The first can be seen as a change in the rate that the subset of the phase space is being browsed whereas the second is a change in the subset itself. 

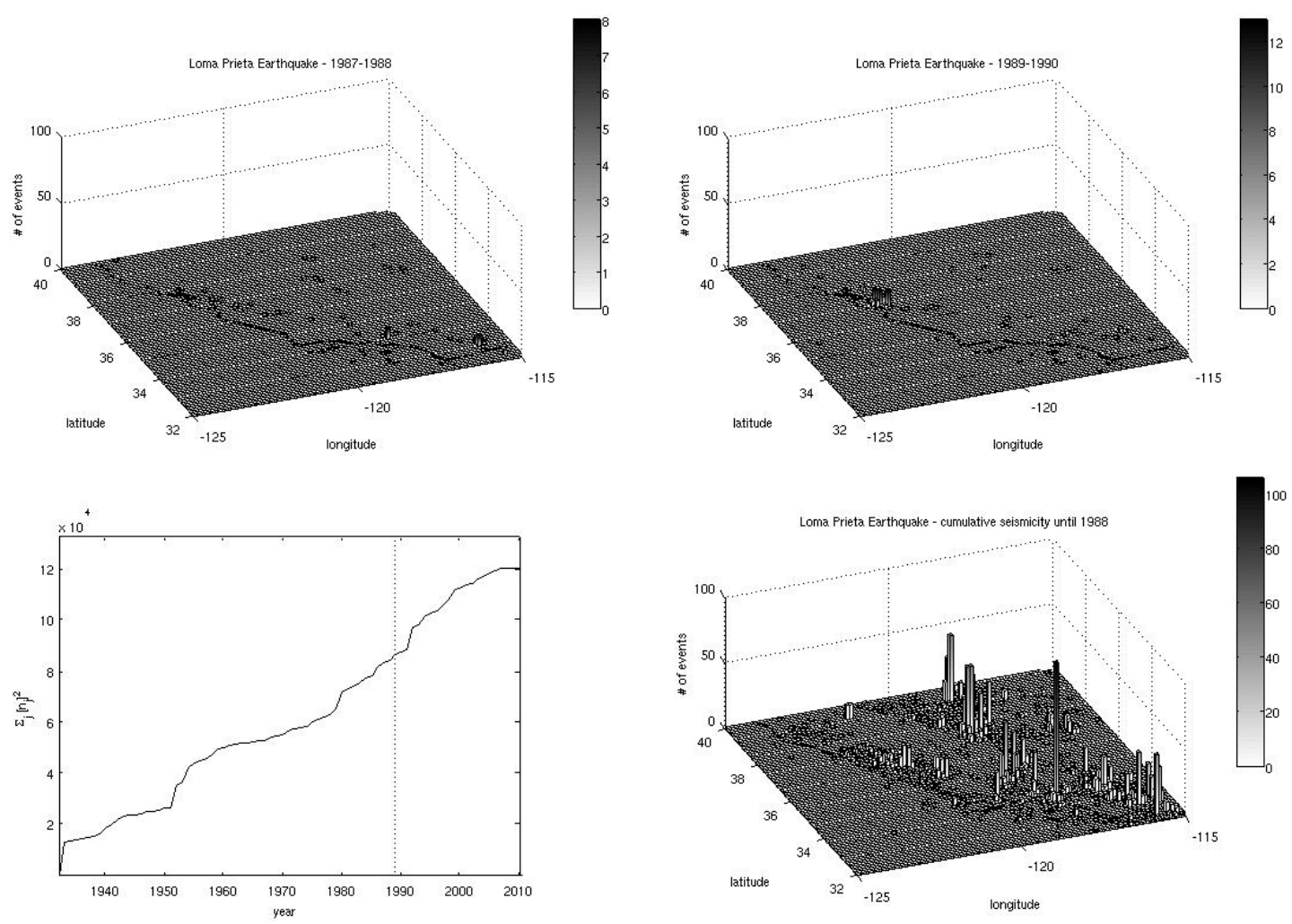

Fig. 6. Distribution of events around the 1989 Loma Prieta earthquake: before the mainshock (top left), after the mainshock (top right) and up until the mainshock since 1932 (bottom right). The approximation in Eq. (4) with the time of occurrence of the 1989 Loma Prieta event as the dashed line are also plotted (bottom left).

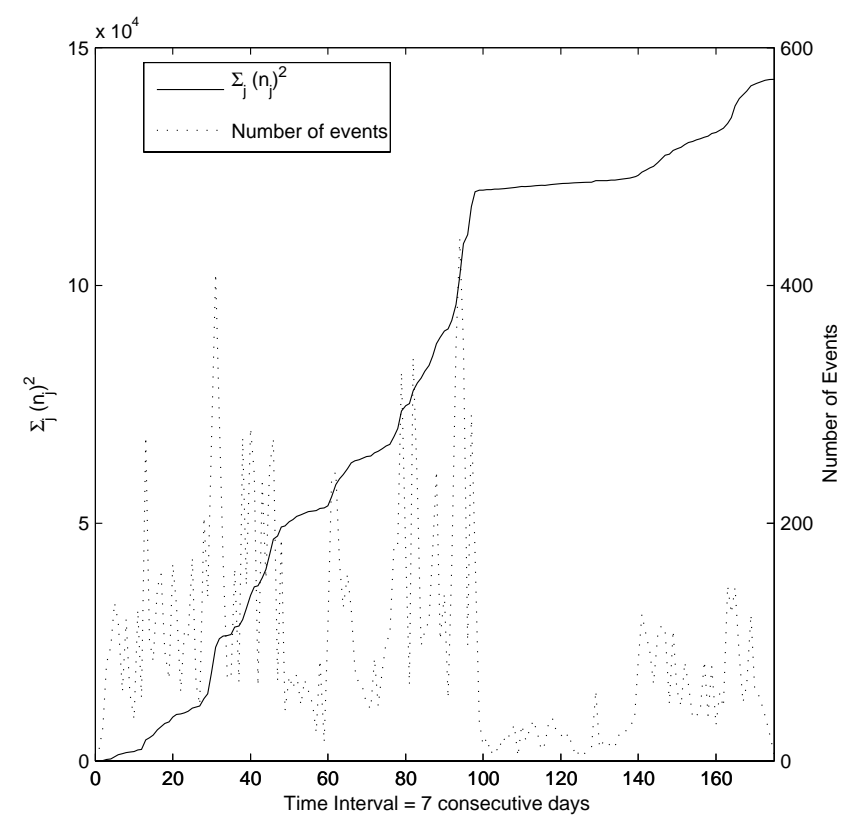

Fig. 7. Plot of Eq. (4) for Kidd Creek D (solid line) and the number of events recorded per time bin $t$ (dashed lines).

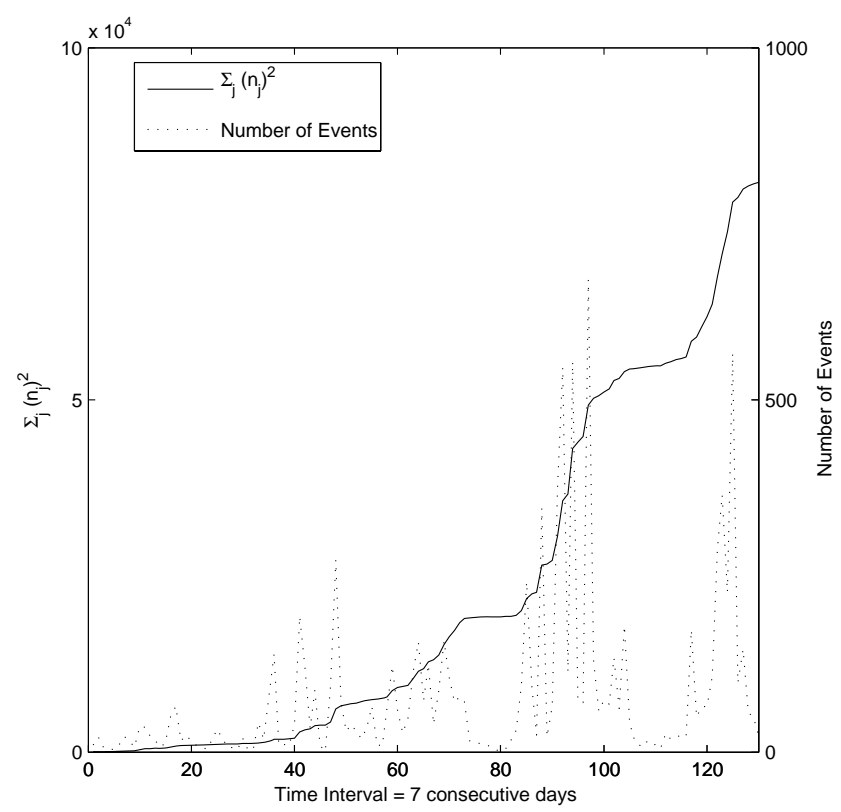

Fig. 8. Plot of Eq. (4) for Macassa mine (solid line) and the number of events recorded per time bin $t$ (dashed lines). 
The implementation of this method to the southern California dataset showed that the disruptions in the effective ergodic periods observed in Tiampo et al. (2007) were due to the aftershock sequences following large earthquakes. It was noticed that these disruptions were highly dependent on the distribution of past seismicity across the region. Large earthquakes such as the 1989 Loma Prieta did not disturb the metric due to the generally lower seismic activity in the region.

Mining seismicity from Kidd Creek D and Macassa mines in Canada were also tested under this interpretation of the TM metric. These mines displayed very different blasting patterns in the periods considered: the first with frequent low-magnitude blasts and the second with rarer larger ones. Regardless of these differences, the same dependence on past seismicity in the analysis was verified in the datasets from both mines. Changes in locations and rates of blasting activity induced changes in seismicity rates in locations with previously low activity, resulting in the constant metric during these periods. It is the same as that which appeared to occur for the 1989 Loma Prieta event and the low seismicity rate in the surrounding local region.

Past studies concerning the application of the TM metric to seismicity have shown that it can be used to highlight well-behaved statistical features of seismicity (Tiampo et al., 2007, 2010). Here another interesting feature of the TM metric is examined and it is shown that the metric provides a simple way to quantify seismicity clustering, and can differentiate between spatial and temporal clustering, given that the region space is chosen carefully. We also demonstrate that its simplicity comes at a cost: the non-uniqueness of the values of the metric, in which different distributions of the cumulative number of events in each box might yield the same score means that various spatial patterns can produce the same value over different time periods. Finally, ongoing studies using simple models of earthquake fault systems suggest that there is a link between the TM results for seismicity that are both unique and more complicated than originally anticipated. Future work will attempt to link the clustering results for both historic data and models of earthquake processes.

\section{Appendix A}

Consider a positive integer $A$ written in terms of $n$ numbers so that

$A=\sum_{i}^{n} a_{i}$

where $a_{i}$ are non-negative real numbers.
From (A1),

$$
\begin{gathered}
A^{2}=\left(\sum_{i}^{n} a_{i}\right)^{2}=\sum_{i}^{n} a_{i}^{2}+\overbrace{\sum_{i ; i \neq j}^{n} a_{i}\left(\sum_{j>i}^{n} a_{j}\right)}^{\geq 0} \\
\sum_{i} a_{i}^{2}=A^{2}-2 \sum_{i ; i \neq j}^{n} a_{i}\left(\sum_{j>i}^{n} a_{j}\right)
\end{gathered}
$$

The extreme values of Eq. (A2) depend on

$f\left(a_{i}\right)=\sum_{i}^{n}\left(a_{i} \sum_{j \neq i}^{n} a_{j}\right)$

The maximum value of Eq. (A2) is obtained when Eq. (A3) is null. Since $a_{i}$ are non-negative integers and $A$ is a positive integer, $f\left(a_{i}\right)=0 \longleftrightarrow a_{i}=\delta_{i j}$, where $\delta_{i j}$ is the Kronecker delta. This means that one value of $a_{i}=A$ and the others are null, i.e. one box contains all events and the others have no events.

The maximum of (A3) can be obtained by using the Lagrange multiplier method using Eq. (A1) as a constraint $g\left(a_{i}\right)=\sum_{i}^{N} a_{i}-A \equiv 0$

$$
\begin{aligned}
\nabla_{a_{i}}\left(f\left(a_{i}\right)-\lambda g\left(a_{i}\right)\right) & =0 \\
\nabla_{a_{i}}\left[\sum_{j ; j \neq i}^{n} a_{j}\left(\sum_{i}^{n} a_{i}\right)\right]-\lambda \nabla_{a_{i}}\left[\sum_{i}^{N} a_{i}-A\right] & =0
\end{aligned}
$$

where $\lambda$ is a Lagrange multiplier.

The evaluation of Eq. (A4) leads to a system of $n$ equations

$\sum_{j \neq i} a_{j}=\lambda, \forall i \in[1, n]$

which results in

$a_{j}=\frac{A}{n}$.

so that $\sum_{j} a_{j}=A$.

The result obtained in Eq. (A6) means that Eq. (A3) is maximized when the values of $a_{j}$ are the same, i.e. for the case in which the events are evenly distributed in the nonempty boxes. The same procedure can be used to one of the $a_{i}$ numbers. It is important to stress that different sets of $a_{i}$ may yield the same $\sum_{i} a_{i}^{2}$.

Acknowledgements. The work presented here was supported by the WSIB Grant for Mining Re-Entry Protocols and the NSERC \& Aon Benfield/ICLR Chair in Earthquake Hazard Assessment. The seismic catalog for southern California was obtained from ANSS (anss.org), while the mining data was obtained under the same WSIB grant.

Edited by: J. Davidsen

Reviewed by: two anonymous referees 


\section{References}

Baiesi, M. and Paczuski, M.: Scale-free networks of earthquakes and aftershocks, Phys. Rev. E, 69, 066106, doi:10.1103/PhysRevE.69.066106, 2004.

de Oliveira, C. R. and Werlang, T.: Ergodic hypothesis in classical statistical mechanics, Rev. Bras. Ensino. Fis., 29(2), 189-201, 2007.

Dieterich, J.: A constitutive law for rate of earthquake production and its application to earthquake clustering, J. Geophys. Res., 99(B2), 2601-2618, 1994.

Farquhar, I. E.: Ergodic theory in statistical mechanics, in: Monographs in Statistical Physics, Volume 7, Inserscience Publishers, 1964.

Ferguson, C. D., Klein, W., and Rundle, J. B.: Spinodals, scaling, and ergodicity in a threshold model with long-range stress transfer, Phys. Rev. E, 60, 1359-1373, 1999.

Felzer, K. R., Becker, T. W., Abercrombie, R. E., Ekström, G., and Rice, J. J.: Triggering of the $1999 M_{W} 7.1$ Hector Mine earthquake by aftershocks of the $1992 M_{W} 7.3$ Landers earthquake, J. Geophys. Res., 107(B9), 2190, doi:10.1029/2001JB000911, 2002.

Gibowicz, S. J. and Kijko, A.: An introduction to mining seismology, Academic New York, 1994.

Helmstetter, A. and Sornette, D.: Subcritical and supercritical regimes in epidemic models of earthquake aftershocks, J. Geophys. Res., 107(B10), 2237, doi:10.1029/2001JB001580, 2002.

Holliday, J. R., Rundle, J. B., Tiampo, K. F., and Turcotte, D. L.: Using earthquake intensities to forecast earthquake occurrence times, Nonlin. Processes Geophys., 13, 585-593, doi:10.5194/npg-13-585-2006, 2006.

Jimenez, A., Tiampo, K. F., Levin, S., and Posadas, A. M.: Testing the persistence in earthquake catalogs: the Iberian peninsula, Europhys. Lett., 73(2), 171-177, 2006.

Kagan, Y. Y. and Jackson, D. D.: Long-term earthquake clustering, Geophys. J. Int., 104, 117-133, 1991.

Kanamori, H.: The nature of seismicity patterns before large earthquakes, in: Series 4: Earthquake Prediction - An International Review, edited by: Ewing, M., AGU Geophys. Mono.: Washington D.C., 1-19, 1981.

Lees, J. M.: Multiplet Analysis at Coso Geothermal, B. Seismol. Soc. Am., 88(5), 1127-1143, 1998.

Mendonza, C. and Hartzell, S. H.: Aftershock patterns and mainshock faulting, B. Seismol. Soc. Am., 78(4), 1438-1449, 1988.

Marsan, D. and Lengliné, O.: Extending earthquakes' reach through cascading, Science, 319, 1076-1079, doi:10.1126/science.1148783, 2008.
Mogi, K.: Earthquake Prediction, Academic Press, New York, 1985.

Mountain, R. D. and Thirumalai, D.: Measures of effective ergodic convergence in liguids, J. Phys. Chem.-US, 93, 69756979, 1989.

Ogata, Y.: Statistical models for earthquake occurrences and residual analysis for point process, J. Am. Stat. Assoc., 83(401), 9-27, 1988.

Reasenberg, P.: Second-order moment of central California seismicity, 1969-1982, J. Geophys. Res., 90(B7), 5479-5495, 1985.

Richardson, E. and Jordan, T. H.: Seismicity in deep gold mines of South Africa: implications for tectonic earthquakes, B. Seismol. Soc. Am., 92(5), 1766-1982, doi:10.1785/0120000226, 2002.

Shcherbakov, R., Yakovlev, G., Turcotte, D. L., and Rundle, J. B.: Model for the distribution of aftershock interoccurrence times, Phys. Rev. Lett., 95, 218501, doi:10.1103/PhysRevLett.95.218501, 2005.

Thirumalai, D. and Mountain, R. D.: Activated dynamics, loss of ergodicity, and transport in supercooled liquids, Phys. Rev. E, 47(1), 479-489, 1993.

Thirumalai, D., Mountain, R. D., and Kirkpatrick, T. R.: Ergodic behavior in supercooled liquids and in glasses, Phys. Rev. A, 39(7), 3563-3573, 1989.

Tiampo, K. F., Klein, W., Li, H. C., Mignan, A., Toya, Y., KohenKadosh, S. Z. L., Rundle, J. B., and Chen, C. C.: Ergodicity and earthquake catalogs: Forecast testing and resulting implications, Pure. Appl. Geophys., published online, doi:10.1007/s00024010-0076-2, 2010.

Tiampo, K. F., Rundle, J. B., Klein, W., Holliday, J., Sá Martins, J. S., and Ferguson, C. D.: Ergodicity in natural earthquake fault networks, Phys. REV. E, 75, 066107, 1-15, doi:10.1103/PhysRevE.75.066107, 2007.

Tiampo, K. F., Rundle, J. B., Klein, W., Sá Martins, J. S., and Ferguson, C. D.: Ergodic dynamics in a natural threshold system, Phys. Rev. Lett., 91(23), 238501, doi:10.1103/PhysRevLett.91.238501, 2003.

Tiampo, K. F., Rundle, J. B., McGinnis, S., Gross, S., and Klein, W.: Mean-field threshold systems and phase dynamics: an application to earthquake fault systems, Europhys. Lett., 60(3), 481487, doi:10.1209/epl/i2002-00289-y, 2002.

Utsu, T.: Statistical features of seismicity, in: International Handbook of Earthquake and Engineering Seismology, Part A, edited by: Lee, W. H. K., Kanamori, H., Jennings, P. C., Kisslinger, C., Academic Press, Amsterdam, 719-732, 2002.

Zaliapin, I., Gabrielov, A., Keilis-Borok, V., and Wong, H.: Clustering Analysis of Seismicity and Aftershock Identification, Phys. Rev. Lett., 101, 018501, doi:10.1103/PhysRevLett.101.018501, 2008. 